\title{
REVIEW ON LIGHT FIDELITY (LI-FI)
}

\author{
Abhishek Sharma ${ }^{1}$ and Mayank Kothari ${ }^{2}$
}

\begin{abstract}
Light fidelity or Li-Fi technology is first proposed by Harald Hass (a German Scientist).Li-Fi is one of the data transmission technique in which radiance or luminance is used a medium for communication between devices. With the help of an LED bulb transmission of data is done by using the fact that variation in its intensity can't match with human persistence of vision. Nowadays the Wi-Fi is the more popular wireless technology that covers within buildings, but we can't ignore the point that Li-Fi is ideal for high speed wireless data transmission though in a meagre area. Li-Fi provides much higher bandwidth, efficiency, availability and security than Wi-Fi. As we know the speed of light is much faster hence we can achieve high speed of data transmission in compared with the one system (Wi-Fi) exists nowadays. This paper aims to traverse this astounding technology and also by comparing the attributes of wireless communication (Wi-Fi) with the light communication (Li-Fi).
\end{abstract}

Keywords - Wireless technology, Light, Electromagnetic spectrum, Optical Communication, Data transfer rate.

\section{INTRODUCTION}

Li-Fi or Light Fidelity is an optical wireless technology which is based on optical wave transmission irrespective of radio waves. This term was coined by pureLiFi co-founder and CSO, German physicist Harald Haas, from the University of Edinburgh. He promoted LI-FI in his 2011 TED Global talk by giving Presentation of an LED light bulb to transmit a data with the speed 10 times faster. As we know speed of light is very high and so will be the data transmission rate through illumination.

In heart of Li-Fi technology are LED bulbs that are used for transmission of data. So seeing future aspects as a replacement of Wi-Fi servers used nowadays, Li-Fi would serve the purpose of Trans receiver which will be fitted with LED lamps that can brighten a room and can dispatch and receive data or information.

As we know transfer of data in today's world is considered as one of the most important activities. So when multiple users access current wireless networks speed tends to reduce and moreover as the number of tools or devices or equipments increases, the limited bandwidth which is available makes it very difficult for the user to utilize or achieve high data transfer rates and uplink or downlink and data. So as to avoid or reduce this reduction in speed, data fetching can be done with the help of light. In the frequency spectrum Li-Fi use the optical spectrum but Wi-Fi uses the radio part of the electromagnetic spectrum.

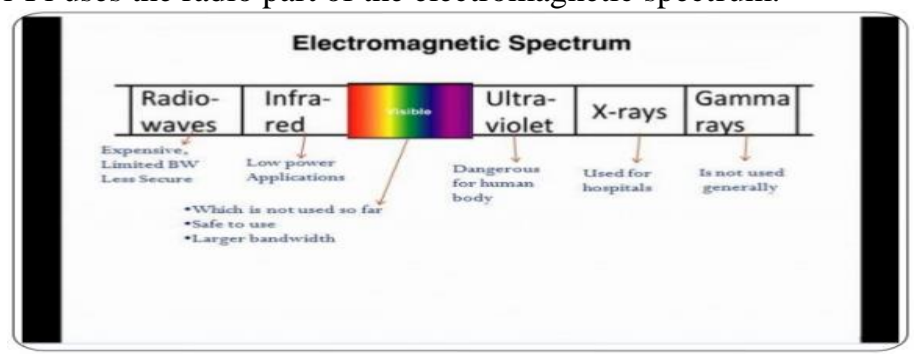

Figure 1. Frequency Spectrum

\footnotetext{
${ }^{1}$ Department of Electronics and communication Engineering, NMIMS University, MPSTME, Shirpur, Maharashtra, India

${ }^{2}$ Department of Electronics and communication Engineering, NMIMS University, MPSTME, Shirpur, Maharashtra, India
} 
Visible light communications (VLC) works by switching LED turn ON and OFF so fast that human eyes cannot detect it. As the amplitude of light is varied, we can detect the changes flickering in light by connecting the LED light circuit with a microchip inside it, thus helping in conversion of light into data in its digital form.

As the light waves cannot penetrate walls and so, this technology is prone from being hacked relative to $\mathrm{Wi}-\mathrm{Fi}$. It is not necessary for Li-Fi to transmit a signal from direct line of sight, lights reflected off the walls can achieve from $70-100 \mathrm{Mbit} / \mathrm{s}$.

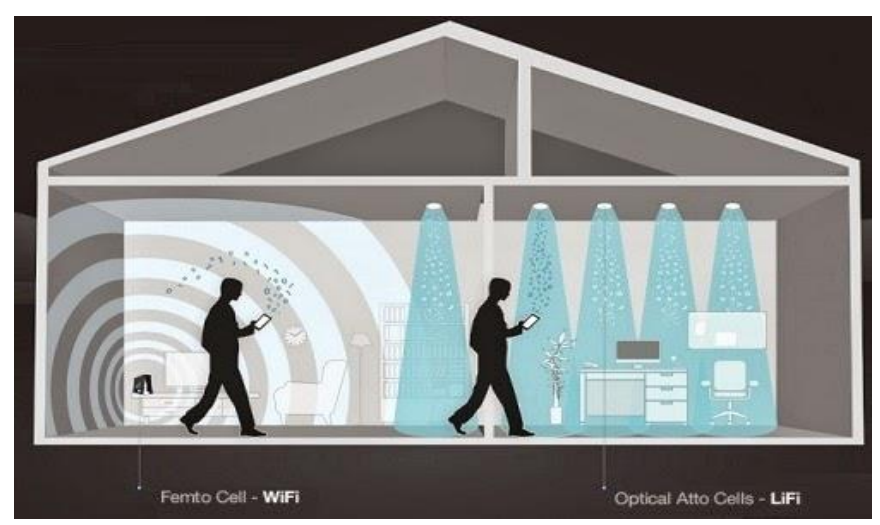

Figure 2. Figure showing difference when a device moves from Wi-Fi to Li-Fi

\section{CONSTRUCTION OF LIFI SYSTEM}

Li-Fi system is based on Visible Light Communications (VLC). It's the next version of Wi-Fi and is very fast and cheap. VLC utilizes visible light between $430 \mathrm{THz}(700 \mathrm{~nm})$ and $770 \mathrm{THz}(390 \mathrm{~nm})$ as optical carrier for data transmission. We can also use other part of spectrum that is ultraviolet or infrared but they pose a threat to humans, so as a result we use faster pulses or visible light to transmit data.

The main components of Li-Fi system are as follows:

a) A luminous LED bulb which can be either fluorescent or incandescent with high brightness, acting as source (transmitter).

b) A photodiode or solar cell to harvest energy which can act as a receiver.

The LED bulb flickers in such a fast rate that human eye can't able to perceive or detect by virtue of its motion. As there are different multiplexing techniques by which rate of data transmission can be increased so by the use of multiple LEDs we can increase the data rate up to $100 \mathrm{Mbps}$, and we can achieve high speed internet sending parallel data or using the technique of parallel data transmission from each LED we can increase visible light communication (VLC). The Li-Fi emitter system consists of 4 primary parts:

a) The plasma generation takes place at the centre of the BULB because of the higher concentration of energy produced in it.

b) The Printed Circuit Board (PCB) houses the microcontroller which is under action to handle different lamp function and control the electrical input and output of the lamp.

c) RF (radio frequency) signal generated by RF power amplifier circuit (PA) is then guided into an electric field about the bulb.

d) And at last all these sub-assemblies are further encompassed or enclosed in an aluminium ENCLOSURE. 


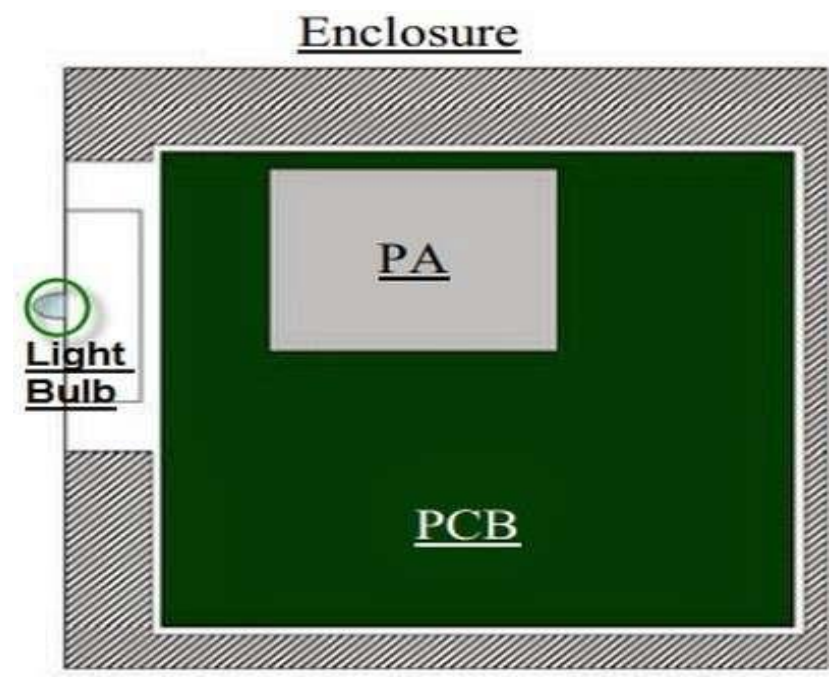

Figure 3. Elements of Li-Fi system

There are several advantages or benefits of this system, which includes

1. High luminosity

2. Brightened colours

3. High luminous power can be achieved which can go up to 150 lumens per watt.

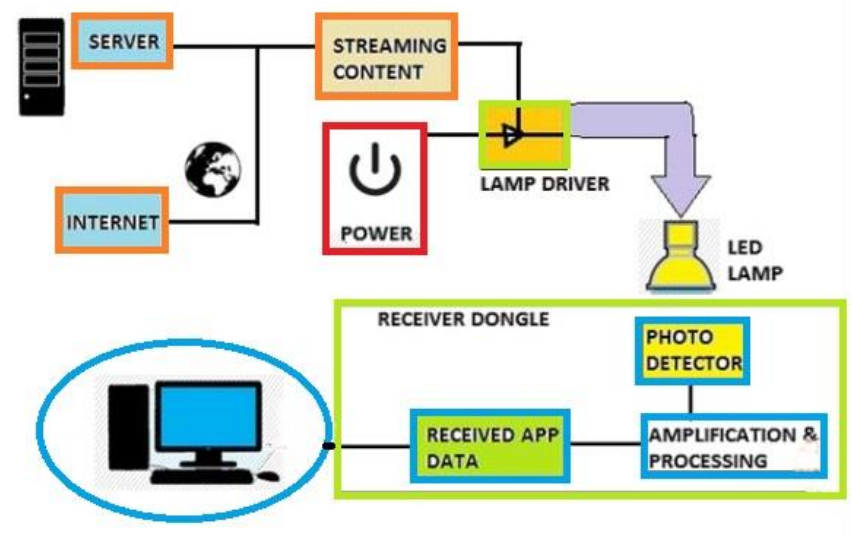

Figure 4. Architecture of Li-Fi

\section{WORKING OF LIFI SYSTEM}

So in Li-Fi we use LED bulbs that can be incandescent or fluorescent flicker, light flickers in such a speed that our human eye can't predict. But there are such receivers or sensors that can detect this flickering and can convert the signal into digital form.

The ceiling-based LED lightening fixture encodes and modulates data messages from the internet as light output at rapid speeds to a receiving photo detector, which converts the changes in light intensity to electrical current. In turn, the electrical current is converted into binary data stream and can be sent to any attached computers and/or mobile devices.

The LED light is switched ON and OFF at a very high rate that human eye can't notice. When LED is switched ON, logic 1 is transmitted and in similar fashion when it'll be switched OFF, logic 0 is transmitted. This LED will flicker the light in such a high speed that human eyes cannot detect it, but we have photodetector to detect such signal. These signals are then converted into electrical current so that ultra-high speed transmission rate can be achieved this speed can go up to 224Gbps. These electrical charges are then converted into a stream of data that can further be transmitted to computer and mobile devices. 
In practical we can implement it easily too. Let's take an example on how we can charge a mobile. as we know a solar cell has the capability of absorbing the light and convert it into electrical energy, this is how we can use a solar cell to charge a mobile phone but now we need to remember that data is encoded in certain changes of the brightness of the LED so if the incoming light fluctuates so does the energy harvested from the solar cell. This means we have a principle mechanism in place to receive information from light and by the solar cell because of the energy fluctuations causes the data to transmit.

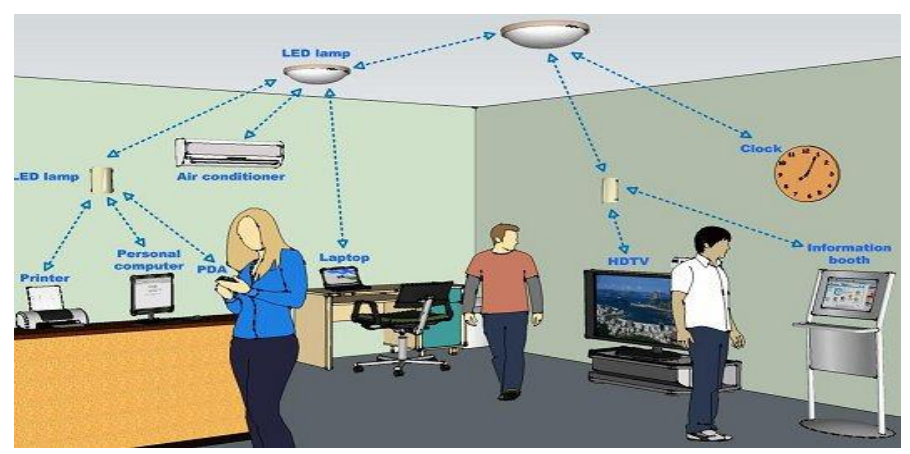

Figure 5. Scenario of an Office where IOT devices working on Li-Fi

\section{COMPARISON BETWEEN LIFI AND WIFI}

$\mathrm{Li}-\mathrm{Fi}$ as the name describes is visible light communication technology applied to access high speed wireless data communication. It's the superset of Wi-Fi. The most important difference between Wi-Fi and Li-Fi is that $\mathrm{Wi}-\mathrm{Fi}$ works on radio frequency and generally its coverage is within the buildings, while Li-Fi is ideal for high density wireless data coverage inside a confined area or room and for relieving radio signal interference issues.

Table 1

\begin{tabular}{|l|l|l|}
\hline Technology & Speed & Data Density \\
\hline Wireless(current) & & \\
& & \\
\hline Wi-Fi IEEE 802.11n & $150 \mathrm{Mbps}$ & $*$ \\
\hline Bluetooth & $3 \mathrm{Mbps}$ & $* *$ \\
\hline IrDA & 4Mbss & $* * *$ \\
\hline Wireless (future) & & \\
& & \\
\hline Wi Gig & 2Gbps & $* *$ \\
\hline Giga-IR & $1 \mathrm{Gbps}$ & $* * *$ \\
\hline Li-Fi & $>1 \mathrm{Gbps}$ & $* * * *$ \\
\hline
\end{tabular}

Table 1 shows the data transfer rate between different existing Wireless technologies, where we can see by means of Li-Fi technology we can achieve astounding data rate as compare to others.

\section{APPLICATIONS OF LIFI}

1. As light waves cannot penetrate through the walls so as a result privacy can be maintained and security can be achieved in a confined area.

2. As we can see use of Light plays a crucial role in Li-Fi so if implemented worldwide every Street Lamp would be a free access point.

3. On aircrafts EMI can be reduced by application of Li-Fi enabled system which allow high data rate connectivity for each passenger, ineffective of sensitive radio equipments.

4. We can use Li-Fi underwater too as light travels faster in water whereas Wi-Fi doesn't works as radio waves gets absorbed in water.

5. $\mathrm{Li}-\mathrm{Fi}$ can also be used in augmented reality as the exhibits in museums and galleries reflect specific lightening henceforth Li-Fi enabled lighting can provide viewer about localised information within the light

6. Li-Fi can also provide car - car communication which allows development of anti-collision systems and exchange of information between drivers while driving. 


\section{ACKNOWLEDGEMENT AND FUTURE SCOPE}

As the future technology is advancing there are numerous possibilities that can be explored. $\mathrm{Li}-\mathrm{Fi}$ is one of the technology if brought into practical implementation, every single bulb or every light source or every Li-Fi lightening can be used somewhat like a Wi-Fi hotspot to transmit wireless data and we will advance towards the eco-friendly future. So the concept of $\mathrm{Li}-\mathrm{Fi}$ is an efficient substitute to radio-based wireless transmission.

With the growing population as number of users are increasing day by day traffic is increasing and congestion makes it difficult for users to gain and transmit data at a faster pace so replacement of wireless radio waves with optical waves is an efficient way to transmit data despite of having reduced bandwidth and more number of users accessing in limited bandwidth. So Li-Fi is one of the best option for accessing internet in a confined space with cheaper cost also in future limitations of artificial light will be overcome for faster transmission.

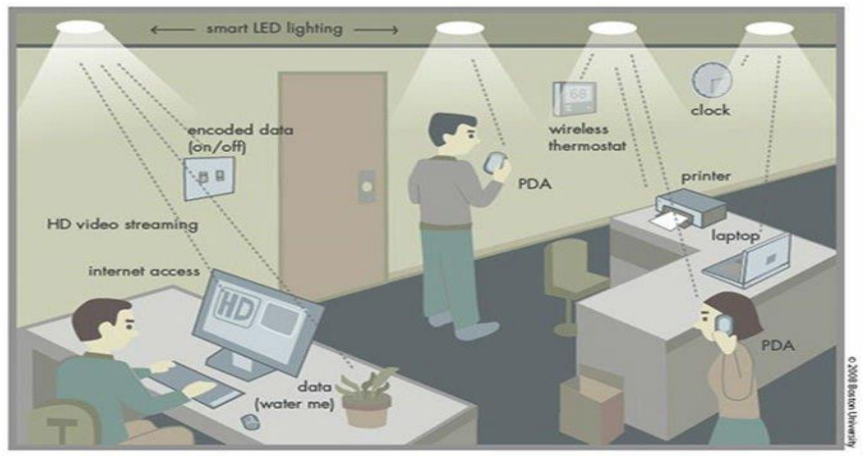

Figure 6. Future working of IOT devices

\section{REFERENCES}

[1.]. Dhakane Vikas Nivrutti, Ravi Ramchandra Nimbalkar, -Light-Fidelity: A Reconnaissance of Future Technologyll, International Journal of Advanced Research in Computer Science and Software Engineering Volume 3, Issue 11, November 2013

[2.]. Navyatha, T.M.Prathyusha, V.Roja, M.Mounika "Li-Fi (Light fidelity)-LED Based Alternative" International Journal of Scientific \& Engineering Research, Volume 4, Issue 5, May-2013

[3.]. Rahul R. Sharma, Raunak, AkshaySanganal-Li-Fi Technology Transmission of data through light IJCTA 2014, ISSN: 2229-6093, Vol 5 (1), 150-154

[4.]. Haas Harald (July 2011). "Wireless data from every light bulb". TED Global. Edinburgh, Scotland.

\section{[5.]. www.ijser.org/researchpaper/A-Review-Paper-on-Li-Fi-Technology}

[6.]. "Li-Fi (Light Fidelity)-The future technology In Wireless communication" by Jyoti Rani, Prema Chauhan, Ritika Tripathi

[7.]. "A Comparative and Critical technical Study of the Li-Fi - (A Future Communication) V/S Wi-Fi" by Prof. (Dr.) Y.P.Singh, Director, KLS Institute of Engineering \& Technology, Chandok, Bijnor U.P.

[8.]. Richard P. Gilliard, Marc DeVincentis, AbdeslamHafidi, Daniel O‘Hare, and Gregg Hollingsworth, -Operation of the LiFi Light Emitting Plasma in Resonant Cavity. 